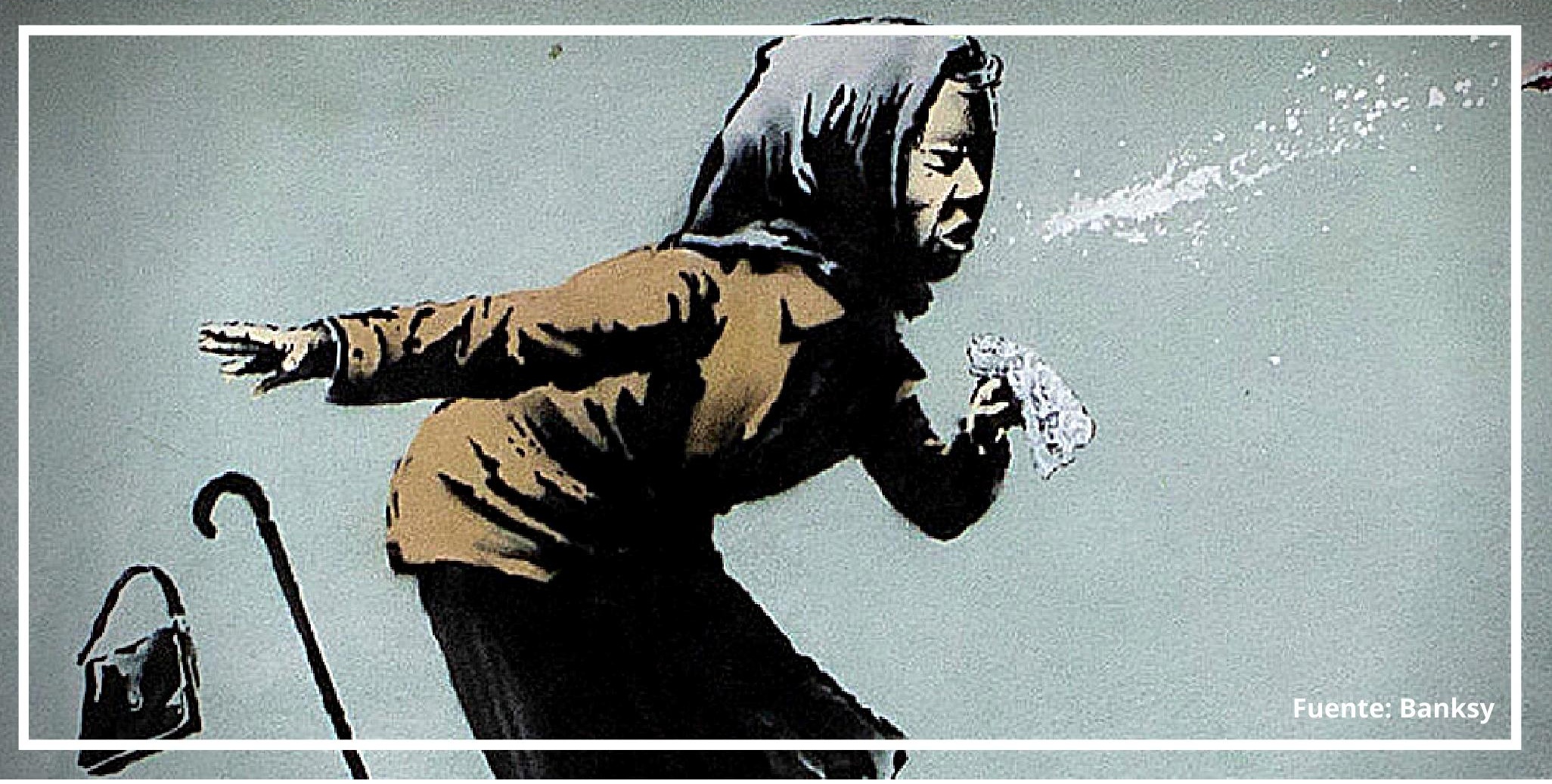

\title{
Sobre el aspecto aerotransportado del coronavirus
}

doi: $10.52749 /$ rh.v1i1.2

\section{ADRIAN DE LA CRUZ}

Miembro de la Sociedad Secular Humanista del Perú (SSH) y del Instituto de Estudios Transhumanistas (IET).

elo.d.hdelacruz@gmail.com

Resumen. Es una opinión ampliamente aceptada que el coronavirus se transmite por contaminación superficial o por contacto cercano de una persona no infectada con una persona infectada. La contaminación superficial usualmente ocurre cuando las gotas de agua infectadas por el exhalar/estornudar/toser de una persona enferma con COVID se depositan en las superficies cercanas. Para frenar esto, la Organización Mundial de la Salud aconseja el distanciamiento social y un buen asesoramiento sobre la higiene de las manos. Se argumenta que el coronavirus también puede transmitirse por el aire en una bocanada cargada con gotas infectadas generadas por una persona enferma con COVID. Un cálculo elemental muestra que una gota respiratoria infectada puede permanecer suspendida alrededor de una hora. Y el consejo de distanciamiento social de por la OMS y por la CDC (Centers for Disease Control and Prevention) puede no ser suficiente en algunas circunstancias como se discute en el texto.

Palabras clave: coronavirus, pandemia, SARS-CoV-2

Cómo citar este artículo:

De la Cruz, A. (2021). Sobre el aspecto aerotransportado del coronavirus. Revista Humanista, 1(1), 12-15. https://doi.org/10.52749/rh.vli1.2

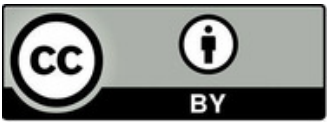




\section{Introducción}

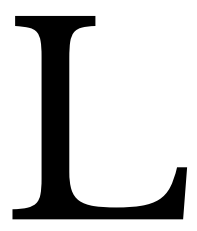

a COVID-19 es una pandemia. Ha infectado a más de $103.111 \mathrm{M}$ de personas en todo el mundo y más de 2.231 M de personas han muerto a causa de ella hasta el 1 de febrero de 2021 (CRC, s.f.). La mayor parte de la información relacionada con la COVID-19 se puede encontrar en el sitio web del OMS (OMS, s.f.) y en el sitio web de los CDC (CDC, s.f.). En la siguiente sección, presentamos un cálculo de la escala de tiempo durante el cual el virus en las gotas de agua infectadas exhaladas por una persona enferma con COVID permanecen en el aire.

2.Un cálculo basado en Newton-Stokes (Despreciando el movimiento browniano)

Sea $h$ la altura típica de un ser humano $(\sim 1.7 \mathrm{~m})$. La exhalación humana genera gotas mucosalivares de tamaños que varían desde 0.5 $\mu m$ a $12 \mu m$ (Yang et al., 2007). Considere la trayectoria de una gota. El tiempo que le toma caer desde la altura $h$ usando mecánica newtoniana despreciando el arrastre del aire está dado por $\sqrt{2 h / g}$, donde $g$ es la aceleración debida a la gravedad. Para $h=1.7 \mathrm{~m}$ este tiempo es aproximadamente $0.6 s$ (menos de un segundo). En este caso, decimos que las gotas no se transportan por el aire y se depositan en las superficies cercanas en un tiempo menor a un segundo.

Pero esta imagen es radicalmente modificada cuando se toma en cuenta el arrastre del aire. La ecuación del movimiento incluyendo la fuerza de arrastre $\left(f_{d}\right)$ para la dirección vertical hacia abajo está dada por

$$
m \frac{d v(t)}{d t}=m g-f_{d} .
$$

Aquí la fuerza de arrastre de Stokes $f_{d}$ está dada por $6 \pi \eta r v . \eta$ es la viscosidad del aire, $r$ es el radio de la gota de agua y $v(t)$ es la velocidad hacia abajo en el tiempo $t$. Considerar que, al tiempo inicial $(t=0)$, la velocidad inicial hacia abajo es cero. La solución de la ecuación anterior inmediatamente da

$$
v(t)=g \tau\left(1-e^{-t / \tau}\right),
$$

donde $\tau=\frac{m}{6 \pi \eta r}$. Y la altura instantánea (medida desde el extremo superior) $v(t)=\frac{d h(t)}{d t}$ está

$$
h(t)=g \tau\left[t+\tau\left(e^{-t / \tau}-1\right)\right] .
$$

Sea el tiempo que toma caer $t_{0}$ y $h\left(t_{0}\right)=h$ la altura típica de una persona.

Consideremos que el radio de una gota de agua típica es de $5 \mu \mathrm{m}$. Su masa será aproximadamente $5,25 \times 10^{-13} \mathrm{Kg}$. Digamos que la temperatura ambiente sea unos $25^{\circ} \mathrm{C}$. La viscosidad del aire a esta temperatura es $\eta=1.85 \times 10^{5} \mathrm{Kg} /(\mathrm{m} . \mathrm{s})$. Al ingresar estos números obtenemos $\tau=\frac{m}{6 \pi \eta r} \simeq 3 \times 10^{-4}$ o $0.3 \mathrm{~ms}$. Para la escala de tiempo (0.6s) más corta (sin arrastre) los exponenciales $\mathrm{e}^{-\mathrm{t} / \mathrm{\tau}}$ en las expresiones de $v(t)$ y $h(t)$ son extremadamente pequeños. Así, $v(t)=v_{\text {terminal }}=g \tau=3 \mathrm{~mm} / \mathrm{s}$ (a partir de la ecuación (2)), que es una velocidad terminal muy baja. Y consideremos que la velocidad terminal se alcanza incluso dentro de una escala de tiempo de milisegundos (ya que $\tau$ está en una escala de tiempo inferior a los ms).

El tiempo total que toma caer desde una altura $h$ es $t_{0}=\frac{h}{g \tau} \simeq 566.7 \mathrm{~s}$, que es alrededor de $9.4 \mathrm{~min}$. En condiciones de viento nulo, las gotas típicas de $5 \mu \mathrm{m}$ de tamaño pueden demorar 9.4 min en asentarse. Las gotas más pequeñas pueden tomar incluso más tiempo. Esta escala de tiempo es inversamente proporcional al cuadrado del radio de la gota:

$$
t_{0}=4.5\left(\frac{\eta h}{g \rho r^{2}}\right)
$$

si consideramos el componente inicial hacia abajo de la velocidad (ya que la exhalación imparte cierta velocidad inicial a las gotas, la respiración nasal generalmente conduce a velocidades iniciales de alrededor de $1.4 \mathrm{~m} / \mathrm{s}$ ), entonces la solución de la ecuación del movimiento conduce a

$$
v(t)=v(0) e^{-t / \tau}+g \tau\left(1-e^{-t / \tau}\right) .
$$

Cualquiera que sea la velocidad inicial, caerá en escalas de tiempo de los milisegundos, ya que $\tau$ es muy pequeña, en escalas de tiempo inferiores a los . Además, cualquier componente transversal de la gota de agua se disipará en escalas de tiempo tan pequeñas. No forma una trayectoria parabólica de proyectil como se visualiza comúnmente. 
3. Situación física importante relacionada con el distanciamiento social

Como una implicación del cálculo anterior, considerar una situación como esta: Considere que las personas están paradas en una fila frente a un cajero automático para retirar dinero en efectivo esperando su turno. También considere que todos están siguiendo la norma de distanciamiento social y se encuentran a $2 \mathrm{~m}$ de distancia el uno del otro. Supongamos que una persona está infectada, pero es asintomática. La persona está parada en su lugar, por más de cinco minutos. Considere también una condición de viento nulo. Esta persona creará una nube invisible cargada de gotas respiratorias de tamaño variado. Estas gotas están todas infectadas. Después de cinco minutos esta persona se adelanta para tomar su turno y una persona detrás de él toma su posición. Tomará unos segundos en avanzar $2 \mathrm{~m}$ hacia adelante. Pero la nube cargada de gotas infecciosas se quedará allí (una gota de $2 \mu \mathrm{m}$ permanecerá suspendida durante aproximadamente una hora y las gotas más pequeñas tardan más en asentarse). Entonces esta persona sana entrará en esa nube infecciosa y puede contraer la infección, incluso si se obedecen las normas de distanciamiento social.

Ahora, si el viento fluye transversalmente a la cola de las personas, eliminará esa nube infecciosa y se dispersará en el aire del ambiente. A distancias suficientemente grandes, la concentración de núcleos de gotas infecciosas será extremadamente baja y ya no será más peligrosa. Pero si el viento fluye a lo largo de la cola, entonces el viento puede transferir esas gotas infecciosas a personas sanas sin protección.

\section{Tiempo de vida de las gotas de agua}

En la discusión anterior se asumió que las gotas de agua preservan su tamaño. Esto no es cierto. Se evaporan en tiempos cortos dependiendo del tamaño de la gota. Sin embargo, una investigación reciente de Bourouiba (2020) muestra que los tiempos de vida son radicalmente modificados ya que las gotas se encuentran en un ambiente muy especial de bocanada respiratoria que contiene muchos vapores de agua. Es muy necesaria una investigación exhaustiva de los tiempos de vida de las gotas y las trayectorias que se tienen en cuenta en este ambiente especial (en la ecua- especial en la ecuación (1) la masa será un parámetro dependiente del tiempo). Pero, cuando una gota infectada se evapora, termina en un núcleo de gota infectada o en un aerosol infectado. Estos son de tamaño submicrométrico y toman aún más tiempo en asentarse. Aunque el enfoque mecánicohidrodinámico anterior no puede aplicarse - ya que la dinámica de los aerosoles será estocástica y el movimiento browniano será importante en su transferencia de un lugar a otro-, le tomará mucho más tiempo asentarse en el suelo. Además, un viento turbulento puede eliminarlo. Esto apunta hacia la naturaleza aerotransportada de la enfermedad. Una discusión acerca del movimiento browniano está dada en la siguiente sección.

\section{Consideración del movimiento browniano}

En la ecuación (1) consideramos el componente sistemático (componente de arrastre $f_{d}$ ) de la fuerza debido al bombardeo de las gotas por las moléculas del aire y despreciamos un componente aleatorio de esta fuerza que también se debe al bombardeo de las gotas por las moléculas del aire [1].

Así, la fuerza total tiene dos componentes: $F(t)=f_{d}+\xi(t) \cdot \xi(t)$ es la fuerza aleatoria cuyo promedio $\langle\xi(t)\rangle=0$, pero correlacionada en el tiempo. La forma más simple de correlación es la correlación delta $\left\langle\xi(t) \xi\left(t^{\prime}\right)\right\rangle=\Gamma \delta\left(t-t^{\prime}\right)$ (Bourouiba, 2020).

Esta fuerza aleatoria puede ser despreciada para gotas muy grandes. Este criterio se basa en el valor del número de Reynolds. La fuerza fluctuante $\xi(t)$ y la fuerza de arrastre $f_{d}$ están intimamente relacionadas entre sí a través del teorema de disipación de fluctuación (ya que ambas se originan a través del bombardeo molecular (Singh, 2020). Así, necesitamos comparar la fuerza de arrastre con otra importante fuerza en el problema de la llamada fuerza inercial. La fuerza inercial es la fuerza requerida para cambiar la cantidad de movimiento de las gotas superior a una distancia de su tamaño $f_{\text {terminal }} \sim m v(v / r)$ (tasa de cambio promedio de la cantidad de movimiento de las gotas superior a su tamaño). La otra fuerza es la fuerza viscosa $\mathrm{f}_{\text {arrastre }}=\eta r v$ (despreciamos los prefactores, ya que estamos interesados en órdenes de magnitud). Su razón se llama número de Reynolds: 


$$
R e=\frac{f_{\text {inercial }}}{f_{\text {arrastre }}}=\frac{\rho r v}{\eta} .
$$

Para gotas de $5 \mu \mathrm{m}$ que se desplazan hacia abajo con una velocidad de $3 \mathrm{~mm} / \mathrm{m}$,-Re . Este es un caso límite. Sin embargo, para el coronavirus (tamaño $\sim 125 \mathrm{~nm}$ ) moviéndose a esta velocidad de deriva, el número de Reynolds resulta ser del orden de 10 . Esto es completamente un régimen dominado por la disipación de fluctuaciones y el virus realizará un movimiento browniano.

Así, las escalas de tiempo obtenidas arriba por el análisis de Newton-Stokes son las escalas de tiempo mínimas posibles (estos son los límites inferiores). Esto es, la gota de $5 \mu \mathrm{m}$ tardará más de 9 min en asentarse. La investigación detallada usando un modelo de Langevin -o más sofisticados- está más allá del alcance de esta investigación.

\section{Consejo al público}

En vista de la naturaleza aerotransportada de la enfermedad hasta cierto punto, se aconseja que cuando alguien está fuera de la casa para obtener productos esenciales en un lugar visitado con frecuencia (como una tienda de comestibles) no solo debe usar una buena mascarilla para protección oral y nasal, sino también se recomienda encarecidamente un equipo de protección para los ojos (protector

\section{Referencias}

Bourouiba, L. (2020). Turbulent Gas Clouds and Respiratory Pathogen Emissions. Potential Implications for Reducing Transmission of COVID-19. JAMA, 323(18), 1837-1838. https://doi.org/10.1001/jama.2020.4756

CDC (Center for Disease Control and Prevention). (s.f.). https://www.cdc.gov./

CRC (Coronavirus Resource Center). (s.f.). Global Map. https://coronavirus.jhu.edu/map.html ocular o protector facial) para cubrir el aspecto aerotransportado de la enfermedad discutido en este trabajo.

\section{Resumen}

Mostramos dentro de la mecánica elemental de Newton-Stokes que las gotas respiratorias no forman una trayectoria parabólica rápida de proyectil como se visualiza comúnmente. Domina el arrastre de Stokes y cae muy lentamente. iUna gota de $5 \mu \mathrm{m}$ tarda alrededor de 9 min en caer desde una altura de $1.7 \mathrm{mn}$ y una gota de $2 \mu \mathrm{m}$ tarda alrededor de una hora en asentarse! Esto muestra claramente la naturaleza aerotransportada de la enfermedad. Incluso cuando se evaporan, terminan formando aerosoles infectados de tamaño submicrométrico que pueden permanecer en el aire durante escalas de tiempo mucho más largas. En vista de los factores anteriores, se deben tomar medidas mucho más precavidas para detener la propagación de este peligroso virus.

[1] Imagínense una película en cámara lenta en la que las gotas son bombardeadas por todos lados por moléculas de aire. En un instante de tiempo, más moléculas bombardearán desde la izquierda y la gota se sacudirá hacia la derecha y en el otro instante de tiempo este desequilibrio se caerá en otra dirección. Esto lleva a una fuerza aleatoria.
Singh, N. (2017). Electronic transport theories. CRC Press. WHO (World Health Organization). (s.f.). WHO Coronavirus (COVID19) Dashboard. https://covid19.who.int/

Yang, S., Lee, G., Chen, C.-M., Wu, C.-C., \& Yu, K.-P. (2007). The size and concentration of droplets generated by coughing in human subjects. Journal of Aerosol Medicine, 20(4), 484-494. https://www.doi.org/10.1089/jam.2007.0610 